$\cdot \frac{33}{10}-28.91$ gs (2)

SSCL-505



Superconducting Super Collider Laboratory

.. il

\title{
LEB Tuner Made out of Titanium Alloy
}

\author{
Y. Goren and B. Campbell
}

September 1991 
SSCL --505

DE92 001931

\title{
LEB Tuner Made Out of Titanium Alloy
}

\author{
Y. Goren and B. Campbell \\ Superconducting Super Collider Laboratory* \\ 2550 Beckleymeade Avenue \\ Dallas, TX 75237
}

September 1991

- Operated by the Cniversities Research Association. Ine. fror the L LS. Department of Energy under Contract No. DF-AC35-89ER-104\%6 
SSCL-505

\title{
LEB Tuner Made Out of Titanium Alloy
}

\author{
Y. Goren and B. Campbell
}

\begin{abstract}
A proposed design of a closed shell tuner for the LEB cavity is presented. The tuner is made out of $\mathrm{Ti}$ alloy which has a high electrical resistivity as well as very good mechanical strength. Using this alloy results in a substantial reduction in the eddy current heating as well as allowing for faster frequency control.
\end{abstract}




\subsection{INTRODUCTION}

One of the most : uportant subjects in the LEB cavity design is the control of the eddy currents developed in its tuner during the frequency sweep. This problem has been solved in the air-cooled Triumf cavity by cutting slots across the tuner, to confine the eddy currents to relatively small areas and reduce the ohmic heating. Such an approach is affronted with a high degree of mechanical complexity in the SSC LEB cavity. Adapting the recommendation of liquid-cooled tuner requires a closed vessel to contain the coolant. We propose to build a closed tuner using the Ti-6Al-4V alloy. Figure 1 is a $3-\mathrm{D}$ description of the tuner. To make use of the high resistivity of this $\mathrm{Ti}$ alloy (two orders of magnitude greater than copper) the maximum eddy current has been reduced from $2700 \mathrm{~A} / \mathrm{cm}^{* *} 2$ to $62 \mathrm{~A} / \mathrm{cm}^{* * 2}$. Using the characteristics of this material in the time domain code PE2D we were able to analyze the eddy current heat dissipation as well as the behavior of the bias magnetic field across the ferrite.

The paper is divided into three sections. The first is an analytical treatment of an infinitely long metallic shell. The second section is a discussion of the numerical results using the time domain PE2D simulation code. The final section is a short summary.

\subsection{EDDY CURRENT IN A METALLIC SHELL}

The geometry of an infinitely long metallic shell is described in Figure 2. Using Ampère's law and split the current into a drive and eddy components we obtain:

$$
\nabla \times B=\mu J+\mu \sigma E
$$

where for the eddy current density we use Ohm's law. The electric and magnetic fields can be expressed in terms of the vector potential $A$ :

$$
\begin{aligned}
& E=-\partial A / \partial t \\
& B=\nabla \times A .
\end{aligned}
$$

Substitute Eq. (2) in Eq. (1) and using the Coulomb gauge we obtain for the infinite shell:

$$
1 / r \partial / \partial r\left(r \partial A_{\theta} /-\partial r\right)-A_{\theta} / r^{2}-\sigma \mu \partial A_{\theta} / \partial t=-\mu J
$$

In regions I and III the solution for $E_{1} .(3)$ is straightforward:

$$
\begin{aligned}
& A_{\theta}^{1}=a_{1} r \\
& A_{\theta}^{\prime \prime l}=a_{2} r+a_{3} / r
\end{aligned}
$$

Where the as are function of time. 


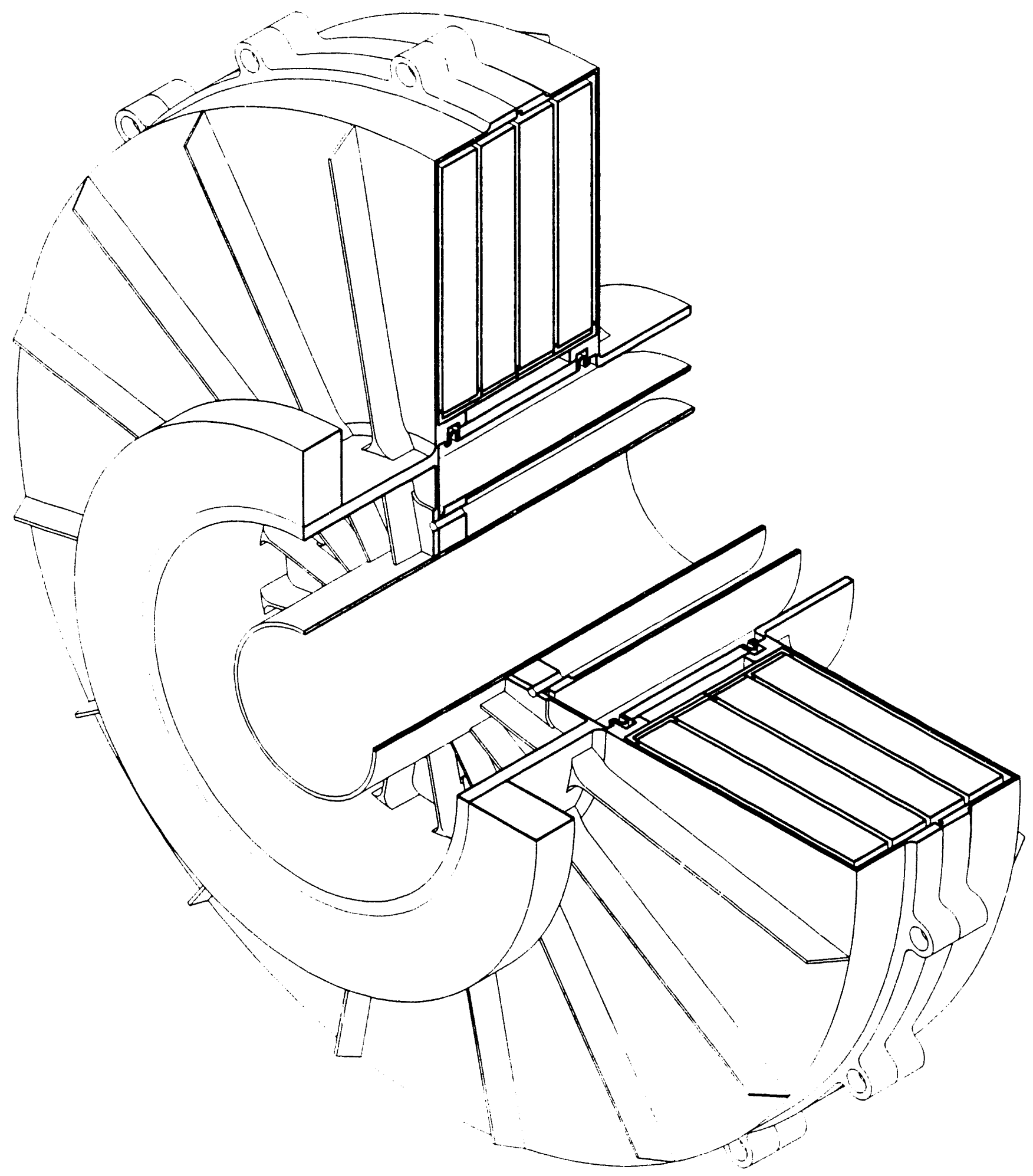

Figure 1. LEB Cavity Design. 


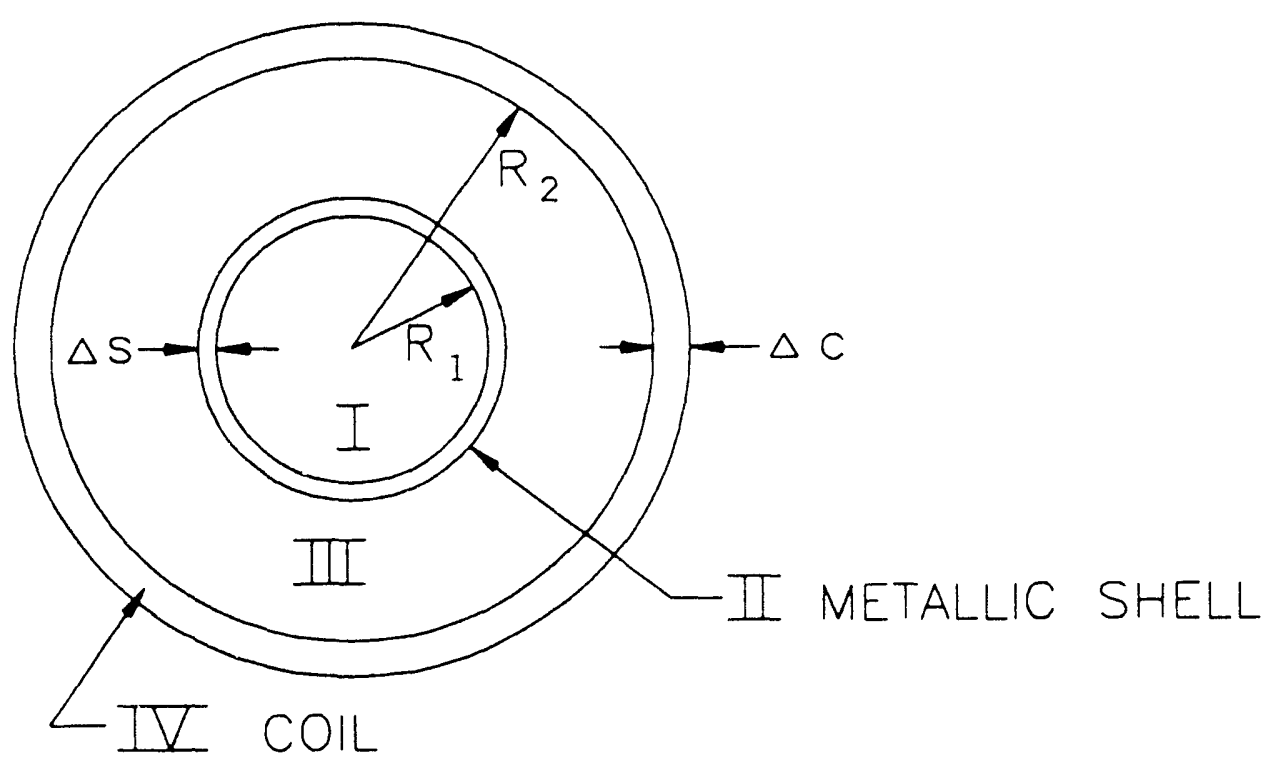

Figure 2. Infinitely Long Metallic Shell in Solenoidal Magnetic Field.

Making the thin shell approximation:

$$
\sigma(r)=\sigma_{0} \Delta_{s} \delta(r-R 1)
$$

where $\sigma_{0}$ is the metal shell conductivity the vector potential becomes continuous across the shell with a discontinuous derivative of the form:

$$
\partial A_{\theta}^{\mathrm{III}} /\left.\partial r\right|_{R 1}-\partial A_{\theta}^{\mathrm{I}} /\left.\partial r\right|_{R 1}=\mu \sigma \Delta_{s} \partial A_{\theta} / \partial t
$$

These boundary conditions determine the relations among the $\alpha$,'s:

$$
\begin{aligned}
& \alpha_{2}=\alpha_{1}+0.5 \mu \sigma \Delta_{s} R_{1} d \alpha_{1} / d t \\
& \alpha_{3}=-0.5 \mu \sigma \Delta_{s} R_{1}^{3} d \alpha_{1} / d t .
\end{aligned}
$$

Assuming spatial homogeneous current density distribution the vector potential in the coil region (region IV) is given by:

$$
A_{\theta}^{I V}=-1 / 3 \mu J r^{2}+\alpha_{4} r+\alpha_{5} / r .
$$

Recuire continuity of $t_{0}$ as well as its first derivative between regions III and IV and put the magnetic field to zero ontside of the coil we end up with a first-order differential 
equation for $\alpha_{1}$ :

$$
0.5 \mu \Delta_{c} J(t)=\alpha_{1}+0.5 \mu \sigma \Delta_{s} R_{1} d \alpha_{1} / d t
$$

Solve Eq. (9) for $\alpha_{1}$ we end up with the vector potential in region I:

$$
A_{\theta}=r Q \delta_{s} \exp \left(-\delta_{s} t\right) \int \exp \left(\delta_{s} \tau\right) J(\tau) d \tau
$$

where we define

$$
\begin{aligned}
Q & =0.5 \mu \Delta_{c} \\
\delta_{s} & =2 /\left(\mu \sigma_{0} \Delta_{s} R_{1}\right) .
\end{aligned}
$$

The eddy current in the metallic shell is given by the time derivative of the vector potential:

$$
J_{\text {eddy }}=-\sigma_{0} \partial A_{\theta} /\left.\partial t\right|_{R 1}=-J \Delta_{c} / \Delta_{s}+\Delta_{c} / \Delta_{s} \delta_{s} \exp \left(-\delta_{s} t\right) \int \exp \left(\delta_{s} \tau\right) J(\tau) d \tau
$$

The magnetic field $B_{z}$ in region $\mathrm{I}$ is given from Eqs. (2) and (10):

$$
B_{z}=1 / r \partial\left(r \cdot A_{\theta}\right) / \partial r=2 Q \delta_{s} \exp \left(-\delta_{s} t\right) \int \exp \left(\delta_{s} \tau\right) J(\tau) d \tau
$$

The parameter $\delta_{s}$ defined in Eq. (10) measures the inverse of the magnetic diffusion time through the metallic shell. For a characteristic time variation of the drive current $T_{\text {ch }}$ we require the inequality $\delta_{s} T_{\mathrm{ch}} \gg 1$ in order to minimize the eddy current in the shell as well as optimize the magnetic time response. To make the last statement more quantitatively let us break the discussion into the two main important aspects namely: the thermal effect of the eddy current in the shell and the magnetic field frequency response.

\subsection{EDDY CURRENT THERMAL EFFECT}

The ohmic heating of the metallic shell by the eddy current can be analyzed by assuming a time profile for the drive current in Eq. (11). Let us assume for implicity a linear growing current density:

$$
J(t)=J_{0} \cdot(t / \tau)
$$

Substitute thin expresson in $E_{1}$. 111 we end after some algebra with the following:

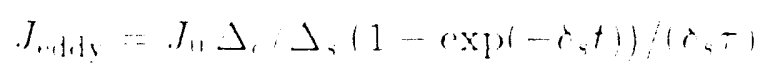


with the maximum eddy current given by:

$$
J_{\max }=J_{0}\left(\mu \sigma_{0} \Delta_{c} R_{1} / 2 \tau\right)
$$

The ratio of the maximum eddy current heating to a copper coil heating is given by the expression:

$$
P_{\text {eddy }} / P_{c}=\left(J_{\max }^{2} / \sigma_{0}\right) /\left(J_{0}^{2} / \sigma_{\mathrm{cu}}\right)=\sigma_{0} \sigma_{\mathrm{cu}}\left(\mu \Delta_{c} R_{1} / 2 \tau\right)^{2}
$$

where $\sigma_{\mathrm{cu}}$ is the copper conductivity.

Require the eddy current heating to be of the same order of magnitude as the coil heating we obtain the following relation for the shell conductivity:

$$
\sigma_{0} \cong\left(2 \tau /\left(\mu \Delta_{c} R_{1}\right)\right)^{2} / \sigma_{\mathrm{cu}}
$$

Substitute in Eq. (17) our geometrical parameters and the typical pulse rise time we end with an estimate of the shell conductivity $\sigma_{0} \cong 5 \times 10^{5} \mathrm{~s} / \mathrm{m}$ which is two orders of magnitude lower than copper conductivity. The Titanium alloy proposed has electrical conductivity of $5.8 \times 10^{5} \mathrm{~s} / \mathrm{m}$ which makes it a good candidate for the LEB tuner.

\subsection{MAGNETIC FIELD FREQUENCY RESPONSE}

The cavity r.f. frequency program is achieved by magnetic biasing the ferrite inside the cavity tuner. The relation between this magnetic field and the current which creates it determines the frequency response of the cavity to deviations from the original frequency program. This response can be analyzed in our simplified model by Fourier decompose of Eq. (12) to obtain:

$$
B_{z}(\omega)=2 Q \delta_{s} J(\omega) /\left(\delta_{s}+i \omega\right)
$$

Using Eq. (18) the bandwidth at the $3 \mathrm{~dB}$ point is determined by:

$$
\Delta_{f}=1 /\left(\pi \mu \sigma_{0} \Delta_{s} R_{1}\right)
$$

which for our geometry and the Titanium alloy conductivity gives:

$$
\Delta_{f}=1.46 \times 10^{3} / \Delta_{4}
$$

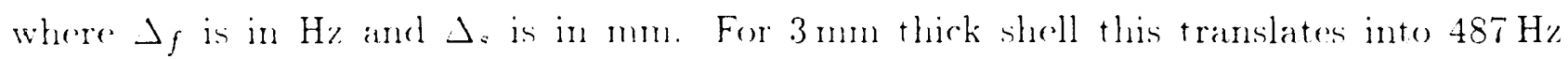
handwidth which exceds the recpuired tuner bandwidth of $100 \mathrm{H} z$. Before moving into the 
numerical analysis discussion it is worth mentioning that this model can be analyzed using the R-L equivalent circuit shown in Figure 3 where the resistivity $R$ and the inductance $L$ are given by:

$$
\begin{aligned}
& R=2 \pi R_{1} /\left(l \Delta_{s} \sigma_{0}\right) \\
& L=\mu \pi R_{1}^{2} / l
\end{aligned}
$$

and $l$ is the axial length of the shell.

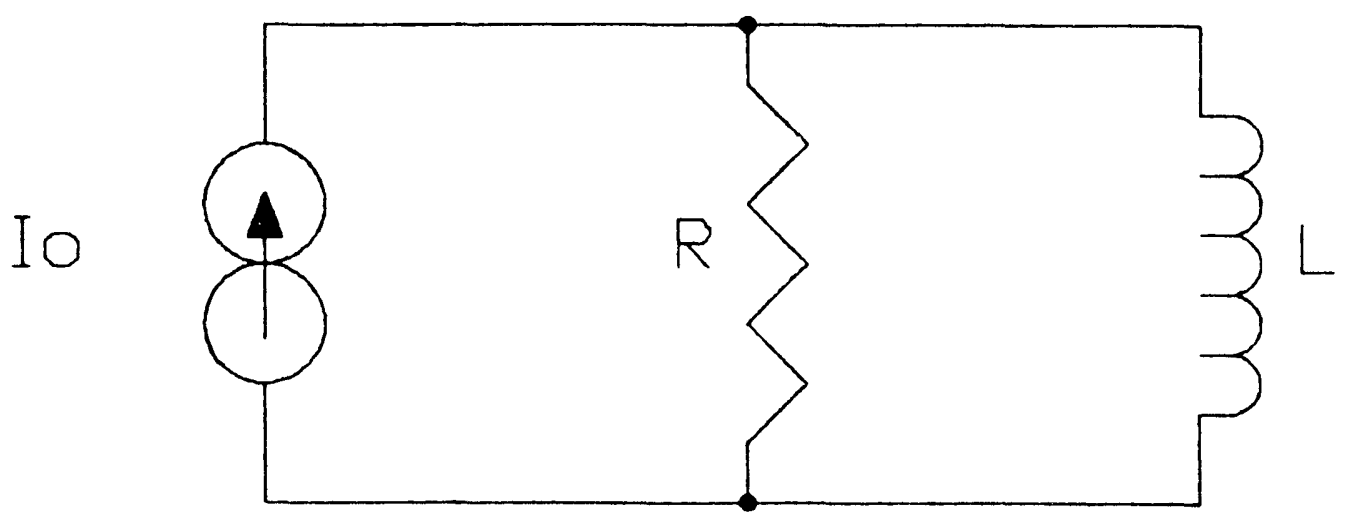

Figure 3. R-L Equivalent Circuit.

\subsection{NUMERICAL ANALYSIS}

Encouraged by the analytical results we numerically simulated the tuner using the PE2D code by Vector Fields Co. PE2D is a two-dimensional time domain electromagnetic code. The code utilizes the finite element method which makes it possible to describe the geometry in fine details. The code is also capable of handling materials with non-linear $\mathrm{B}-\mathrm{H}$ curves. The tuner geometry is shown in Figure 4 where the scale is in $\mathrm{cm}$. The drive current vs. time has been determined in two stages. At first the code was run in the steady state mode with various currents to establish a relation between the drive current and r.f. permeability. Knowing the r.f. permeability has a function of time from the given frequency program, the drive current vs. time is determined to first order. In the second stage this time behavior has been corrected for the eddy currents effect using the code to its full capability of transient analysis with non-linear materials. Figure 5 describes the current program for one full cycle of the LEB. The current reaches about 17,000 amp.turn at $50 \mathrm{msec}$, then drops in $30 \mathrm{msec}$ to about 4000 amp.-turn and stays there to the end of the cycle. The maximum eddy current is developed at the top of the tuner where its two parts are clumped (see Figure 4). Figure 6 describes the absolute value of the eddy current at this point as at function of tine. The deviations from a smooth curve are related to the way the code handles the derivative of the drive current in Figure 5 . 
The maximum eddy current density obtained is $62 \mathrm{amp} / \mathrm{cm}^{* * 2}$ at $17 \mathrm{msec}$ from start of the cycle. In comparison the eddy curreni for a copper framed tuner is about $2700 \mathrm{amp} / \mathrm{cm}^{* *} 2$ for the same driving current program. Substitute the maximum time derivative of the drive current in Eq. (15) for the maximum eddy current developed in our infinitely long metallic shell; we end with $J_{\text {eddy }}=58.8 \mathrm{amp} / \mathrm{cm}^{* *} 2$ which is very close to the simulation resuits of $62 \mathrm{amp} / \mathrm{cm}^{* *} 2$. The thermal power averaged on a cycle developed at this point is about $0.18 \mathrm{w} / \mathrm{cm}^{* *} 3$ which can be handled without much difficulty by the tuner internal coolant. The equi-magnetic field lines across the tuner at $t=0.017 \mathrm{sec}$ of maximum eddy current development are shown in Figure 7. No distortions in the magnetic field lines are observed. In comparison Figure 8 presents the magnetic lines distribution for the case of a copper tuner where strong distortions can be observed. To quantify the effect of the eddy currents on the behavior of the magnetic field we plot in Figure 9 the magnetic field distribution along the ferrites. The upper curve describes the magnetic field as a function of the radius for a tuner with no eddy currents. The lower curve describes the magnetic field in our proposed tuner. Both curves are plotted at the time $t=0.017 \mathrm{sec}$ of maximum eddy currents. The difference is most pronounced at lower radiuses and reaches maximum of $2.8 \%$ at $14 \mathrm{~cm}$. This difference in magnetic fields results in a difference in the r.f. frequency of about $140 \mathrm{KHz}$. A slight modification in the tuner drive current program is needed to compensate for this difference. 


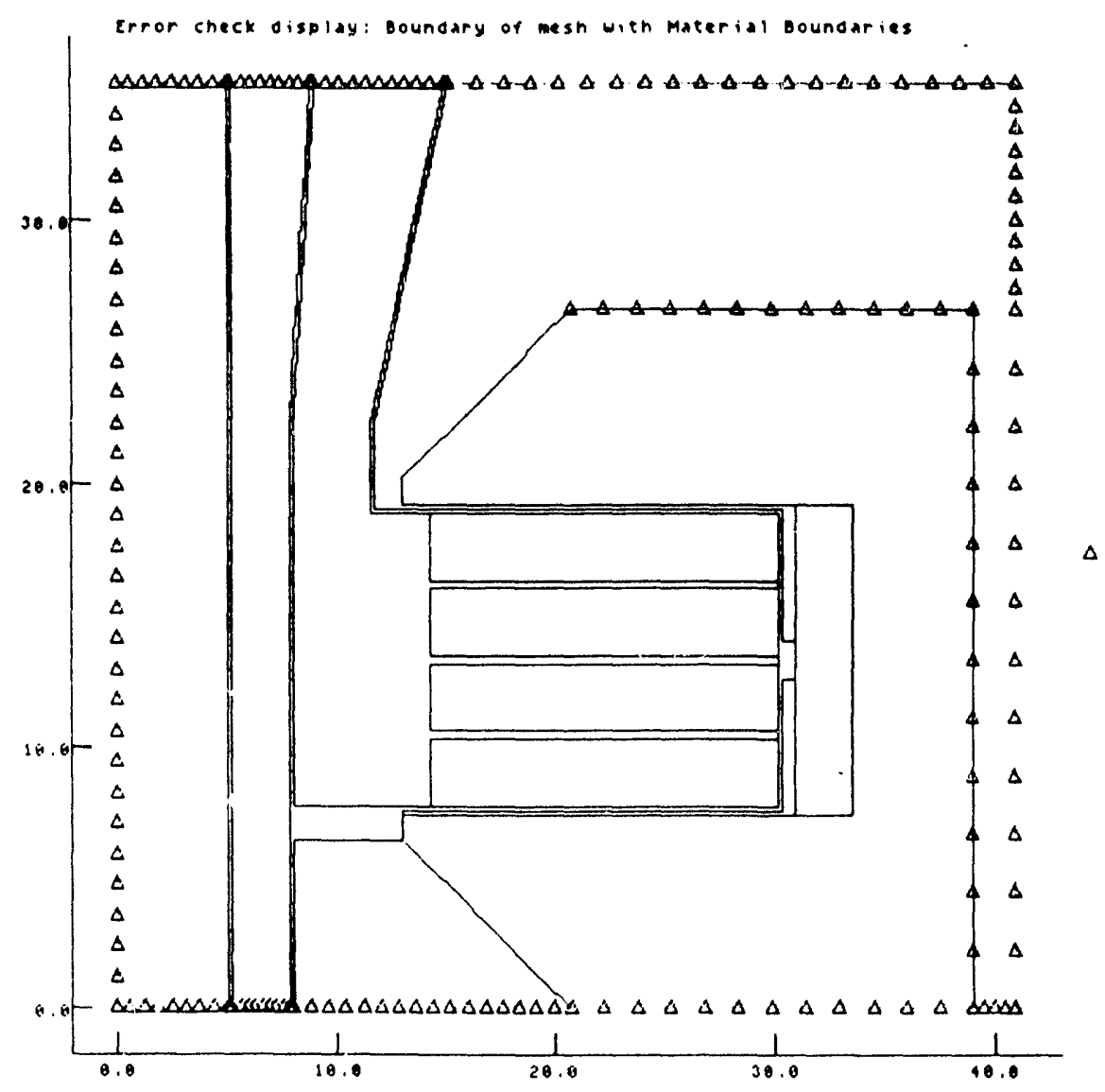

Figure 4. Tuner Geography for PE2D.

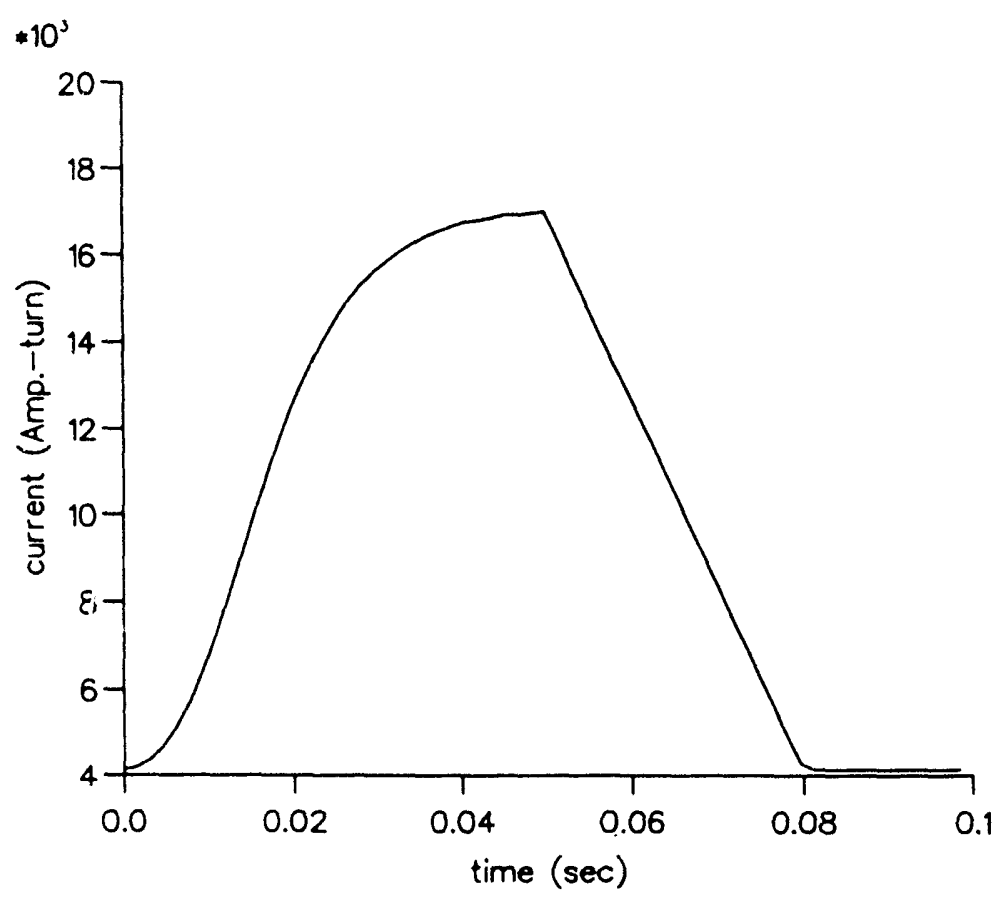

Figure 5. Ampere-Tur: in LEB Tuner. 


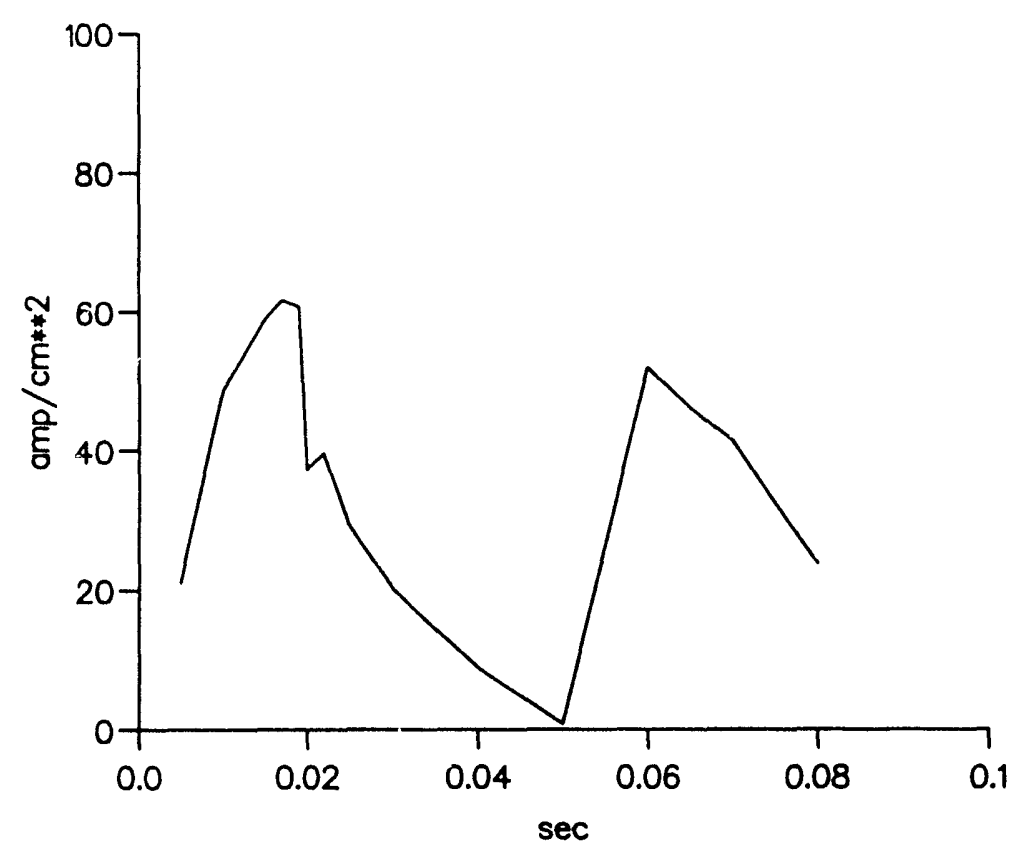

Figure 6. Eddy Current in Titanium Tuner.

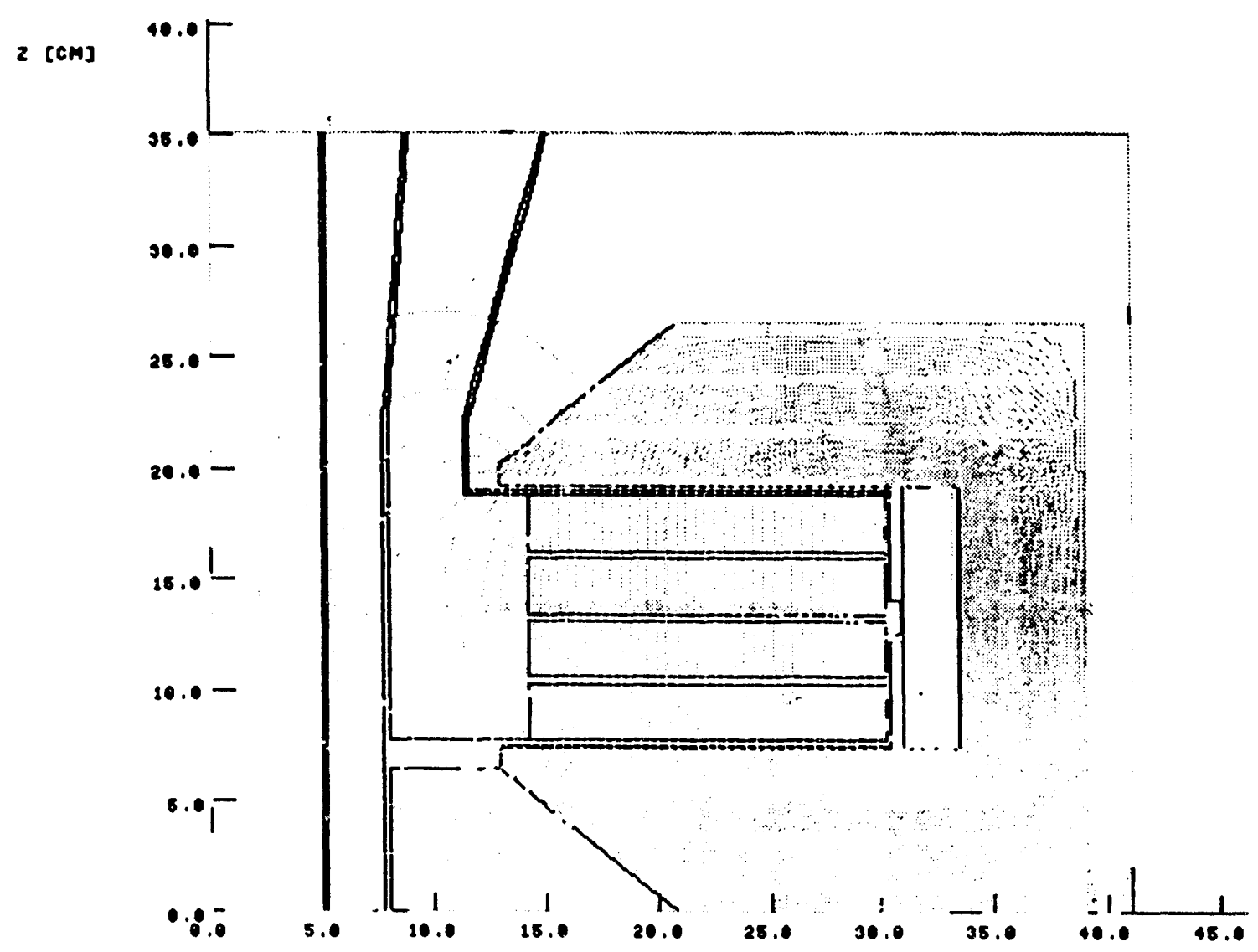

Figure 7. Magnetic Field Lines at $t=0.017$ seconds. 


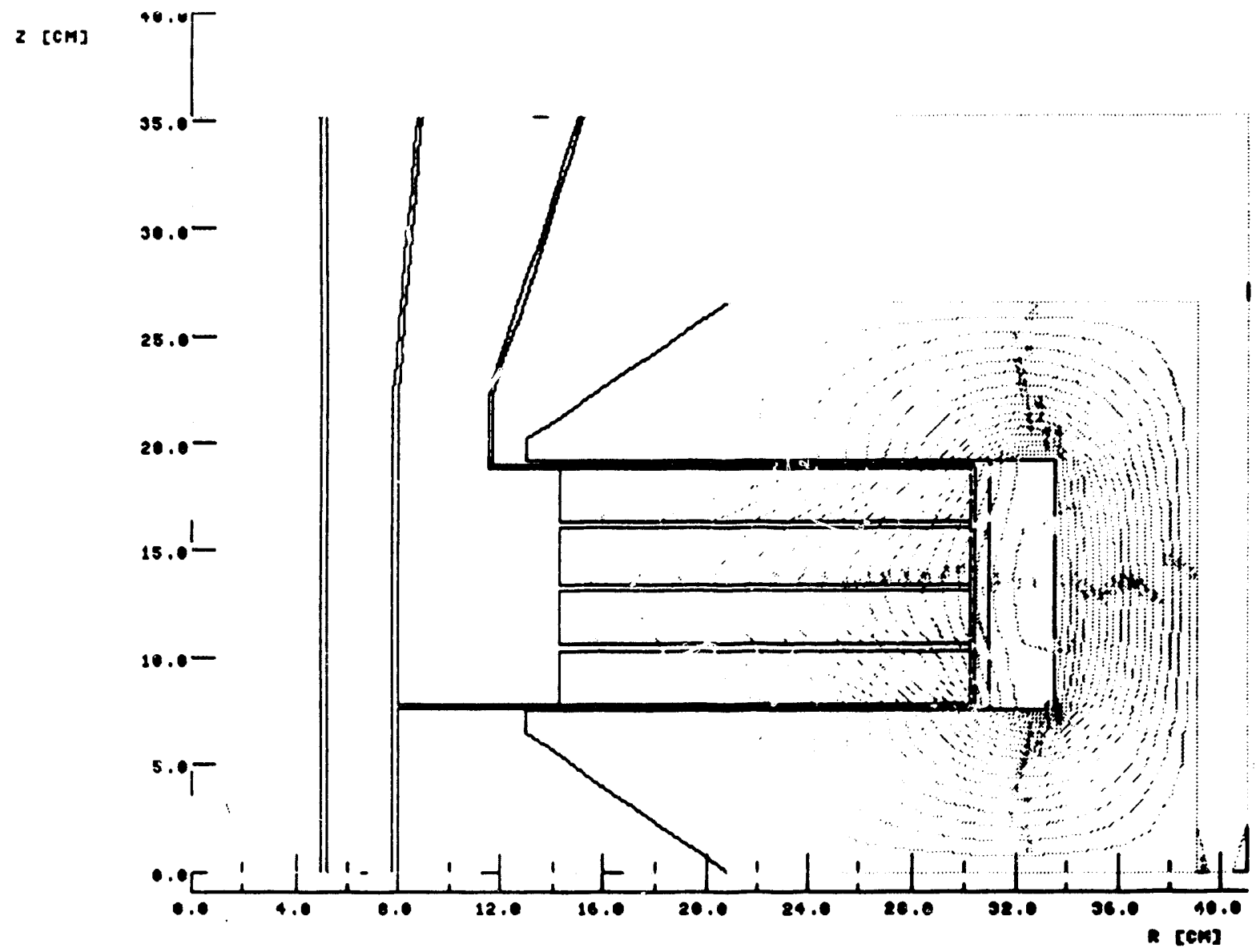

Figure 8. Copper Tuner Magnetic Line Distribution.

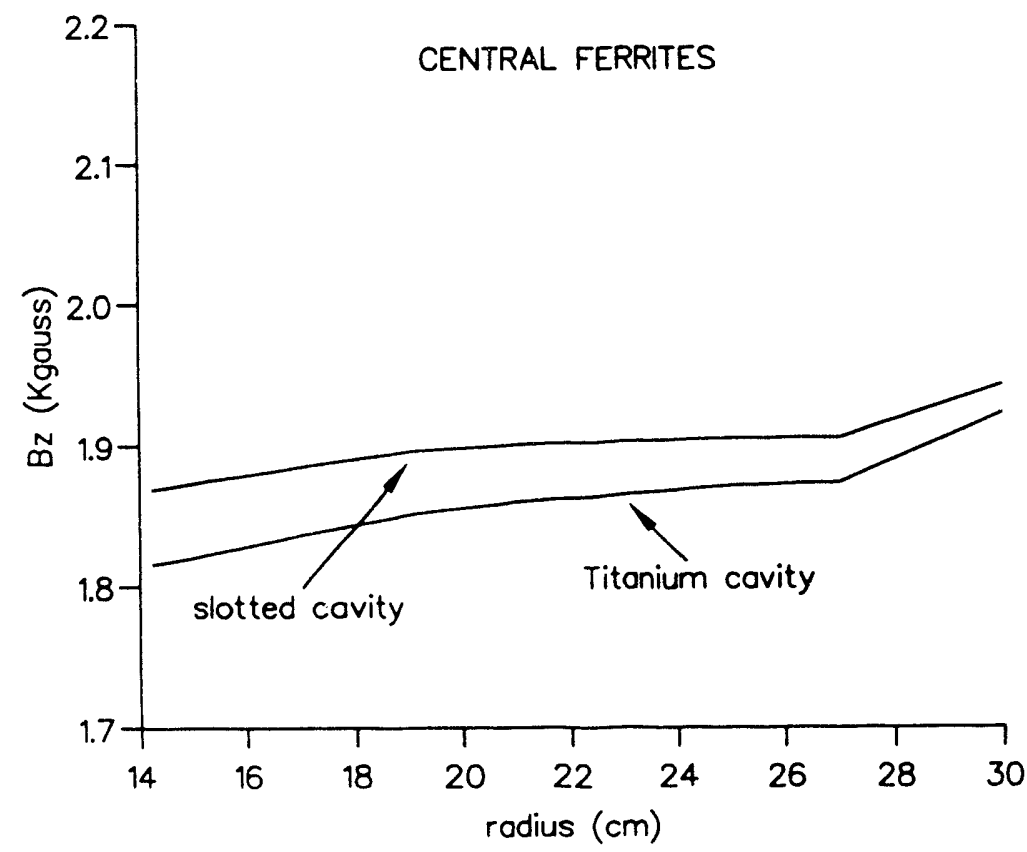

Figure 9. Magnetic: Field Homogeneity $(t=0.017 \mathrm{sec})$. 


\subsection{SUMMARY}

Analytical as well as numerical simulation results are indicating that a closed shell tuner for the LEB cavity is realizable. In order to reduce the eddy current effects the shell has to be made out of electrically lossy material. That material should have at least two orders of magnitude higher resistivity than copper. The best candidate we found is a Ti alloy which has the required electrical characteristics, good mechanical tensile strength and acceptable thermal conductivity. 
Disclaimer Notice

This report was propared as an account of work sponsored by an aponcy of the Unied States Government. Norther the Unied Stales Government or any agoncy thereot, noe any of their omoloyeos. makes any warranty. oxpress or implied, or assumes any hoal liability or reaponeibility for the accuracy, comploteness, or usoluiness $\alpha$ any information. apparatus. product. or process disclosed, or reprosents that its use would not inisinge pivately owned rights. Peterence herein to any specitic commerctal product. procoss, or sernce by trade name, tradernark manufacturer, or atherwse. does not nocessarly constifute of imply is ondorsernent. recommendation, or favoing by the Unito States Government or any agency thoreot. The veows and opinions of authors expressed herein do not nocessarity stale or reflect those of the Uniiz-t States Governmont or any agoncy theroot

Superconducting Super Collider Laboratory is an equal opportunity employer. 

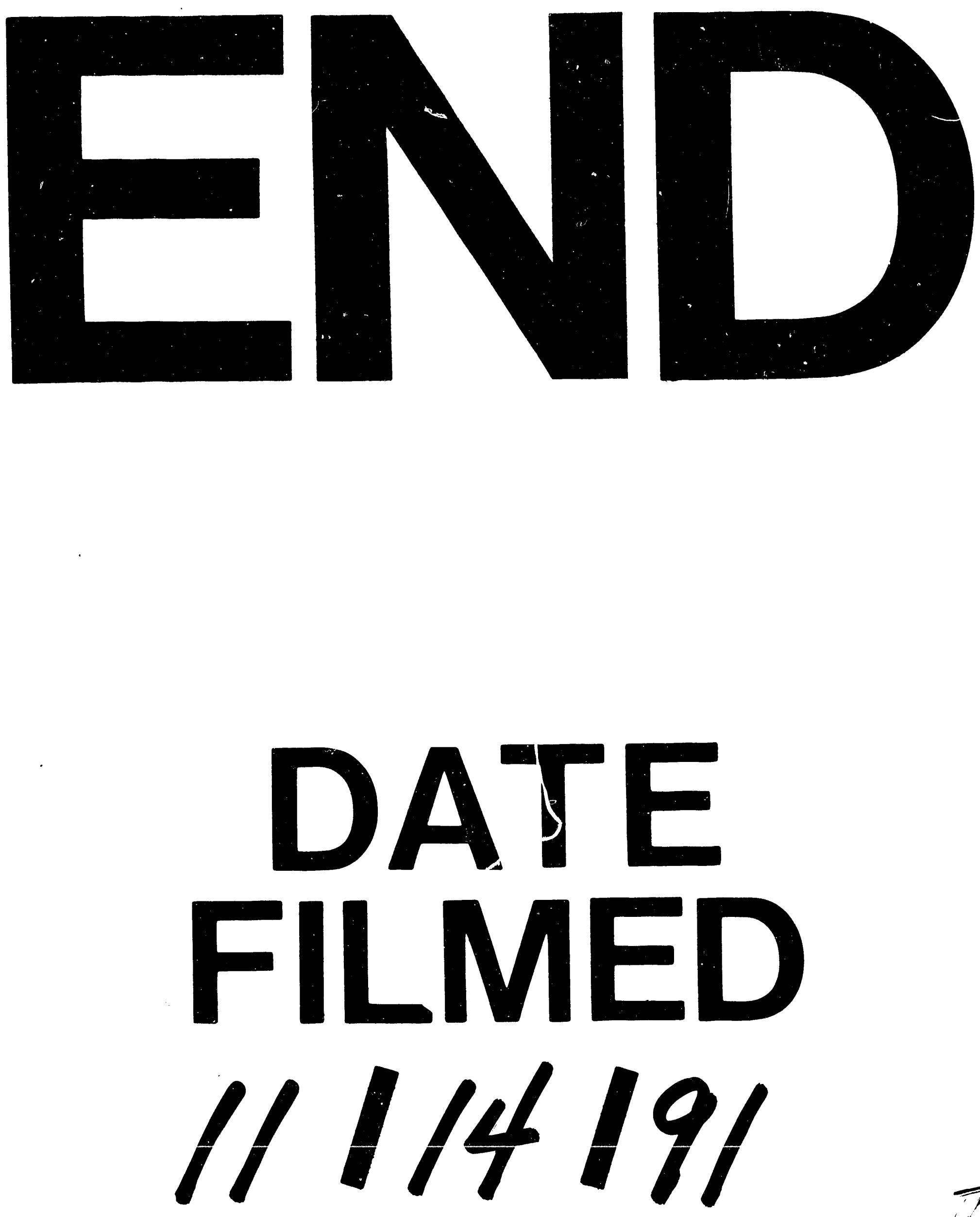

$\overline{1}$ 
